\title{
EXISTENCE OF MILD SOLUTIONS FOR FRACTIONAL EVOLUTION EQUATIONS
}

\author{
YONG ZHOU, LU ZHANG and XIAO HUI SHEN
}

Communicated by Juan J. Trujillo

\begin{abstract}
In this paper, we study the nonlocal Cauchy problems of fractional evolution equations with RiemannLiouville derivative by considering an integral equation which is given in terms of probability density. By using the theory of Hausdorff measure of noncompactness, we establish various existence theorems of mild solutions for the Cauchy problems in the cases $C_{0}$ semigroup is compact or noncompact.
\end{abstract}

1. Introduction. In this paper, we assume that $X$ is a Banach space with the norm $|\cdot|$. Let $a \in \mathbf{R}^{+}, J=[0, a]$ and $J^{\prime}=(0, a]$. Denote $C(J, X)$ as the Banach space of continuous functions from $J$ into $X$ with the norm $\|x\|=\sup _{t \in[0, a]}|x(t)|$, where $x \in C(J, X)$, and $B(X)$ are the spaces of all bounded linear operators from $X$ to $X$ with the norm $\|Q\|_{B(X)}=\sup \{|Q(x)|:|x|=1\}$, where $Q \in B(X)$ and $x \in X$.

Consider the following nonlocal Cauchy problem of fractional evolution equation with Riemann-Liouville derivative

$$
\left\{\begin{array}{l}
\left({ }^{L} D_{0+}^{q} x\right)(t)=A x(t)+(F x)(t) \quad \text { almost all } t \in[0, a], \\
\left(I_{0+}^{1-q} x\right)(0)+g(x)=x_{0},
\end{array}\right.
$$

where ${ }^{L} D_{0+}^{q}$ is the Riemann-Liouville derivative of order $q, I_{0+}^{1-q}$ is the Riemann-Liouville integral of order $1-q, 0<q<1, A$ is the

2010 AMS Mathematics subject classification. Primary 26A33, 34A08, 35R11.

Keywords and phrases. Fractional evolution equations, integral equations, Riemann-Liouville derivative, mild solutions, $C_{0}$ semigroup, measure of noncompactness.

Project supported by the National Natural Science Foundation of P.R. China (No. 11271309), the Specialized Research Fund for the Doctoral Program of Higher Education (No. 20114301110001) and Hunan Provincial Natural Science Foundation of China (No. 12JJ2001).

Received by the editors on February 14, 2013, and in revised form on March 3, 2013.

DOI:10.1216/JIE-2013-25-4-557 Copyright (C)2013 Rocky Mountain Mathematics Consortium 
infinitesimal generator of a strongly continuous semigroup of bounded linear operators (i.e., $C_{0}$ semigroup) $\{Q(t)\}_{t \geq 0}$ in Banach space $X$, $F: C(J, X) \rightarrow L(J, X)$ is a given (non-linear) operator, $g: C(J, X) \rightarrow$ $L(J, X)$ is a given operator satisfying some assumptions and $x_{0}$ is an element of the Banach space $X$.

Problem (1) can be viewed as a generalization of the following problem

$$
\left\{\begin{array}{l}
\left({ }^{L} D_{0+}^{q} x\right)(t)=A x(t)+f(t, x(t)), \quad \text { almost all } t \in[0, a], \\
\left(I_{0+}^{1-q} x\right)(0)+g(x)=x_{0},
\end{array}\right.
$$

where $f: J \times X \rightarrow X$ is a given function satisfying some assumptions. Indeed, under suitable conditions, the following operator

$$
(F x)(t)=f(t, x(t)), \quad \text { for } t \in[0, a]
$$

maps $C(J, X)$ into $L(J, X)$. Thus, problem (1) reads as (2).

The existence of mild solutions for fractional evolution equations with Caputo derivative has been considered in several recent papers (for instance, see $[\mathbf{6}, \mathbf{8}, \mathbf{1 0}, \mathbf{1 5}, \mathbf{1 8}, \mathbf{1 9}]$ and the references therein), much less is known about the fractional evolution equations with RiemannLiouville derivative. In most of the existing articles (see for instance $[6,8,15,18,19]$ and the references therein), Schauder's fixed point theorem, Schaefer's fixed point theorem, Krasnoselskii's fixed point theorem, Darbo's fixed point theorem, or the Kuratowski measure of noncompactness are employed to obtain fixed points of the solution operator of problem (1) under some restrictive conditions.

In this paper, by using the theory of Hausdorff measure of noncompactness, we study nonlocal Cauchy problem (1) in the case where $Q(t)$ is compact or noncompact. The next section introduces some notations and useful concepts from fractional calculus and the theory of measure of noncompactness. In Section 3, we obtain the appropriate definition on mild solutions of problem (1) by considering an integral equation which is given in terms of probability density. Section 4 is devoted to the study of various existence theorems of mild solutions for problem (1). Finally, an example is given for demonstration.

2. Preliminaries. In this section, we introduce preliminary facts which are used throughout this paper. 
First, we recall some basic definitions and properties of the fractional calculus. For more details, see Kilbas, Srivastava and Trujillo [7].

Definition 2.1 [7]. The fractional integral $I_{0+}^{q} f$ of order $q$ for a function $f \in A C[0, \infty)$ is defined as

$$
\left(I_{0+}^{q} f\right)(t)=\frac{1}{\Gamma(q)} \int_{0}^{t} \frac{f(s)}{(t-s)^{1-q}} d s, \quad t>0,0<q<1,
$$

provided the right side is point-wise defined on $[0, \infty)$, where $\Gamma(\cdot)$ is the gamma function.

Definition $2.2[7]$. Riemann-Liouville derivative ${ }^{L} D_{0+}^{q} f$ of order $q$ for a function $f \in A C[0, \infty)$ can be written as

$$
\left({ }^{L} D_{0+}^{q} f\right)(t)=\frac{1}{\Gamma(1-q)} \frac{d}{d t} \int_{0}^{t} \frac{f(s)}{(t-s)^{q}} d s, \quad t>0,0<q<1 .
$$

If $f$ is an abstract function with values in $X$, then integrals which appear in Definitions 2.1 and 2.2 are taken in Bochner's sense.

Next, we recall some definitions and properties of the measure of noncompactness.

The measure of noncompactness $\alpha$ is said to be:

(i) Monotone if, for all bounded subsets $B_{1}, B_{2}$ of $X, B_{1} \subseteq B_{2}$ implies $\alpha\left(B_{1}\right) \leq \alpha\left(B_{2}\right)$;

(ii) Nonsingular if $\alpha(\{x\} \cup B)=\alpha(B)$ for every $x \in X$ and every nonempty subset $B \subseteq X$;

(iii) Regular $\alpha(B)=0$ if and only if $B$ is relatively compact in $X$.

One of the most important examples of measure of noncompactness is the Hausdorff measure of noncompactness $\alpha$ defined on each bounded subset $B$ of $X$ by

$$
\alpha(B)=\inf \left\{\varepsilon>0: B \subset \bigcup_{j=1}^{m} B_{\varepsilon}\left(x_{j}\right) \text { where } x_{j} \in X\right\},
$$

where $B_{\varepsilon}\left(x_{j}\right)$ is a ball of radius $\leq \varepsilon$ centered at $x_{j}, j=1,2, \ldots, m$. 
It is well known that the Hausdorff measure of noncompactness $\alpha$ enjoys the above properties (i)-(iii) and other properties (see $[\mathbf{1}, \mathbf{3}, \mathbf{5}$, 9]).

(iv) $\alpha\left(B_{1}+B_{2}\right) \leq \alpha\left(B_{1}\right)+\alpha\left(B_{2}\right)$, where $B_{1}+B_{2}=\{x+y: x \in$ $\left.B_{1}, y \in B_{2}\right\}$

(v) $\alpha\left(B_{1} \cup B_{2}\right) \leq \max \left\{\alpha\left(B_{1}\right), \alpha\left(B_{2}\right)\right\}$;

(vi) $\alpha(\lambda B) \leq|\lambda| \alpha(B)$ for any $\lambda \in \mathbf{R}$;

For any $W \subset C(J, X)$, we define

$$
\int_{0}^{t} W(s) d s=\left\{\int_{0}^{t} u(s) d s: u \in W\right\}, \quad \text { for } t \in[0, a],
$$

where $W(s)=\{u(s) \in X: u \in W\}$.

Proposition 2.1. If $W \subset C(J, X)$ is bounded and equicontinuous, then $\overline{c o} W \subset C(J, X)$ is also bounded and equicontinuous.

Proposition 2.2 [12]. If $W \subset C(J, X)$ is bounded and equicontinuous, then $t \rightarrow \alpha(W(t))$ is continuous on $J$, and

$$
\begin{aligned}
\alpha(W) & =\max _{t \in J} \alpha(W(t)), \\
\alpha\left(\int_{0}^{t} W(s) d s\right) & \leq \int_{0}^{t} \alpha(W(s)) d s, \quad \text { for } t \in[0, a] .
\end{aligned}
$$

Proposition 2.3 [13]. Let $\left\{u_{n}\right\}_{n=1}^{\infty}$ be a sequence of Bochner integrable functions from $J$ into $X$ with $\left\|u_{n}(t)\right\| \leq \widetilde{m}(t)$ for almost all $t \in J$ and every $n \geq 1$, where $\widetilde{m} \in L\left(J, \mathbf{R}^{+}\right)$. Then the function $\psi(t)=\alpha\left(\left\{u_{n}(t)\right\}_{n=1}^{\infty}\right)$ belongs to $L\left(J, \mathbf{R}^{+}\right)$and satisfies

$$
\alpha\left(\left\{\int_{0}^{t} u_{n}(s) d s: n \geq 1\right\}\right) \leq 2 \int_{0}^{t} \psi(s) d s .
$$

The map $\mathscr{T}: B_{0} \subseteq Y \rightarrow Y$ is said to be an $\alpha$-contraction if there exists a positive constant $k<1$ such that $\alpha(\mathscr{T}(B)) \leq k \alpha(B)$ for any bounded closed subset $B \subseteq B_{0}$. 
To prove our main results, we need the following lemmas associated with Hausdorff measure of noncompactness.

Lemma 2.1. Let $B$ be a closed and convex subset of a Banach space $Y$. Suppose $\mathscr{T}: B \rightarrow B$ is a continuous operator and $\mathscr{T}(B)$ is bounded. For each bounded subset $B_{0} \subset B$, set

$$
\mathscr{T}^{1}\left(B_{0}\right)=\mathscr{T}\left(B_{0}\right), \mathscr{T}^{n}\left(B_{0}\right)=\mathscr{T}\left(\overline{\mathrm{co}}\left(\mathscr{T}^{n-1}\left(B_{0}\right)\right)\right), \quad n=2,3, \ldots
$$

If there exist a constant $0 \leq k<1$ and a positive integer $n_{0}$ such that, for any bounded subset $B_{0} \subset B$,

$$
\alpha\left(\mathscr{T}^{n_{0}}\left(B_{0}\right)\right) \leq k \alpha\left(B_{0}\right)
$$

then there exists a $D \subset B$ such that $\alpha(\mathscr{T}(D))=0$.

The proof of Lemma 2.1 is similar to that of Lemma 2.4 in [11], and it is thus omitted.

Lemma 2.2 (Darbo-Sadovskii's fixed point theorem) [1]. If $B$ is a bounded, closed and convex subset of a Banach space $Y$, and the continuous map $\mathscr{T}: B \rightarrow B$ is an $\alpha$-contraction, then the map $\mathscr{T}$ has at least one fixed point in $B$.

\section{Definition of mild solutions.}

Definition 3.1 [12]. The Wright function $M_{q}(\varrho)$ is defined by

$$
M_{q}(\varrho)=\sum_{n=1}^{\infty} \frac{(-\varrho)^{n-1}}{(n-1) ! \Gamma(1-q n)}, \quad 0<q<1, \varrho \in \mathbf{C} .
$$

It is known that $M_{q}(\varrho)$ satisfies the following equality (see [17])

$$
\int_{0}^{\infty} \theta^{\delta} M_{q}(\theta) d \theta=\frac{\Gamma(1+\delta)}{\Gamma(1+q \delta)}, \text { for } \delta \geq 0 .
$$

Lemma $3.1[\mathbf{1 6}]$. (i) Let $\xi, \eta \in \mathbf{R}$ be such that $\eta>-1$. If $t>0$, then

$$
\left(I_{0+}^{\xi} \frac{s^{\eta}}{\Gamma(\eta+1)}\right)(t)=\left\{\begin{array}{ll}
\left(t^{\xi+\eta}\right) / \Gamma(\xi+\eta+1) & \text { if } \xi+\eta \neq-n \\
0 & \text { if } \xi+\eta=-n
\end{array},\left(n \in \mathbf{N}^{+}\right) .\right.
$$


(ii) Let $\xi>0$ and $\varphi \in L((0, a), X)$. Define

$$
G_{\xi}(t)=I_{0+}^{\xi} \varphi, \text { for } t \in(0, a)
$$

Then

$$
\left(I_{0+}^{\eta} G_{\xi}\right)(t)=\left(I_{0+}^{\xi+\eta} \varphi\right)(t), \quad \eta>0, \text { almost all } t \in[0, a] .
$$

Lemma 3.2. Assume that the operator $F: C\left(J^{\prime}, X\right) \rightarrow L\left(J^{\prime}, X\right)$ is continuous. The nonlocal Cauchy problem (1) is equivalent to the integral equation

$$
\begin{aligned}
x(t)= & \frac{t^{q-1}}{\Gamma(q)}\left(x_{0}-g(x)\right) \\
& +\frac{1}{\Gamma(q)} \int_{0}^{t}(t-s)^{q-1}[A x(s)+(F x)(s)] d s, \quad \text { for } t \in(0, a] .
\end{aligned}
$$

Proof. Suppose (3) is true. Then

$$
\begin{aligned}
\left(I_{0+}^{1-q} x\right)(t)=\left(I_{0+}^{1-q}\right. & {\left[\frac{s^{q-1}}{\Gamma(q)}\left(x_{0}-g(x)\right)\right.} \\
& \left.\left.+\frac{1}{\Gamma(q)} \int_{0}^{s}(s-\tau)^{q-1}[A x(\tau)+(F x)(\tau)] d \tau\right]\right)(t),
\end{aligned}
$$

and applying Lemma 3.1 we obtain that $\left(I_{0+}^{1-q} x\right)(t)=x_{0}-g(x)+\int_{0}^{t}[A x(s)+(F x)(s)] d s, \quad$ almost all $t \in[0, a]$.

This proves that $\left(I_{0+}^{1-q} x\right)(t)$ is absolutely continuous on $[0, a]$. Since $F \in L\left(J^{\prime}, X\right)$, then we have

$$
\left({ }^{L} D_{0+}^{q} x\right)(t)=\frac{d}{d t}\left(I_{0+}^{1-q} x\right)(t)=A x(t)+(F x)(t), \quad \text { almost all } t \in[0, a]
$$


and

$$
\left(I_{0+}^{1-q} x\right)(0)+g(x)=x_{0} .
$$

The proof of the converse is given as follows.

Suppose (1) is true. Then

$$
\left(I_{0+}^{q-1}\left({ }^{L} D_{0+}^{q} x\right)\right)(t)=\left(I_{0+}^{q-1}(A x(s)+(F x)(s))\right)(t) .
$$

Since

$$
\begin{aligned}
\left(I_{0+}^{q-1}\left({ }^{L} D_{0+}^{q} x\right)\right)(t) & =x(t)-\frac{t^{q-1}}{\Gamma(q)}\left(I_{0+}^{1-q} x\right)(0) \\
& =x(t)-\frac{t^{q-1}}{\Gamma(q)}\left(x_{0}-g(x)\right), \quad \text { for } t \in(0, a],
\end{aligned}
$$

then we have

$$
\begin{aligned}
x(t)= & \frac{t^{q-1}}{\Gamma(q)}\left(x_{0}-g(x)\right)+\left(I_{0+}^{q-1}(A x(s)+(F x)(s))\right)(t) \\
=\frac{t^{q-1}}{\Gamma(q)}\left(x_{0}-g(x)\right)+ & \frac{1}{\Gamma(q)} \int_{0}^{t}(t-s)^{q-1}[A x(s)+(F x)(s)] d s, \\
& \quad \text { for } t \in(0, a] .
\end{aligned}
$$

The proof is complete.

Before giving the definition of the mild solution of (1), we firstly prove the following lemma.

\section{Lemma 3.3. If}

$$
\begin{aligned}
x(t)= & \frac{t^{q-1}}{\Gamma(q)}\left(x_{0}-g(x)\right) \\
& +\frac{1}{\Gamma(q)} \int_{0}^{t}(t-s)^{q-1}[A x(s)+(F x)(s)] d s, \quad \text { for } t>0
\end{aligned}
$$

holds, then we have

$x(t)=t^{q-1} P_{q}(t)\left(x_{0}-g(x)\right)+\int_{0}^{t}(t-s)^{q-1} P_{q}(t-s)(F x)(s) d s, \quad$ for $t>0$, 
where

$$
P_{q}(t)=\int_{0}^{\infty} q \theta M_{q}(\theta) Q\left(t^{q} \theta\right) d \theta
$$

Proof. Let $\lambda>0$. Applying the Laplace transform $\nu(\lambda)=\int_{0}^{\infty} e^{-\lambda s} x(s) d s \quad$ and $\omega(\lambda)=\int_{0}^{\infty} e^{-\lambda s}(F x)(s) d s$, for $\lambda>0$ to (4), we have

$$
\begin{aligned}
\nu(\lambda)= & \frac{1}{\lambda^{q}}\left(x_{0}-g(x)\right)+\frac{1}{\lambda^{q}} A \nu(\lambda)+\frac{1}{\lambda^{q}} \omega(\lambda) \\
= & \left(\lambda^{q} I-A\right)^{-1}\left(x_{0}-g(x)\right)+\left(\lambda^{q} I-A\right)^{-1} \omega(\lambda) \\
= & \int_{0}^{\infty} e^{-\lambda^{q} s} Q(s)\left(x_{0}-g(x)\right) d s \\
& +\int_{0}^{\infty} e^{-\lambda^{q} s} Q(s) \omega(\lambda) d s
\end{aligned}
$$

provided that the integrals in (5) exist, where $I$ is the identity operator defined on $X$.

Set

$$
\psi_{q}(\theta)=\frac{q}{\theta^{q+1}} M_{q}\left(\theta^{-q}\right)
$$

whose Laplace transform is given by

$$
\int_{0}^{\infty} e^{-\lambda \theta} \psi_{q}(\theta) d \theta=e^{-\lambda^{q}}, \quad \text { where } q \in(0,1)
$$

Using (6), we get

$$
\begin{aligned}
\int_{0}^{\infty} & e^{-\lambda^{q} s} Q(s)\left(x_{0}-g(x)\right) d s \\
& =\int_{0}^{\infty} q t^{q-1} e^{-(\lambda t)^{q}} Q\left(t^{q}\right)\left(x_{0}-g(x)\right) d t \\
& =\int_{0}^{\infty} \int_{0}^{\infty} q \psi_{q}(\theta) e^{-(\lambda t \theta)} Q\left(t^{q}\right) t^{q-1}\left(x_{0}-g(x)\right) d \theta d t
\end{aligned}
$$




$$
\begin{aligned}
& =\int_{0}^{\infty} \int_{0}^{\infty} q \psi_{q}(\theta) e^{-\lambda t} Q\left(\frac{t^{q}}{\theta^{q}}\right) \frac{t^{q-1}}{\theta^{q}}\left(x_{0}-g(x)\right) d \theta d t \\
& =\int_{0}^{\infty} e^{-\lambda t}\left[q \int_{0}^{\infty} \psi_{q}(\theta) Q\left(\frac{t^{q}}{\theta^{q}}\right) \frac{t^{q-1}}{\theta^{q}}\left(x_{0}-g(x)\right) d \theta\right] d t
\end{aligned}
$$

(8)

$$
\begin{aligned}
\int_{0}^{\infty} & e^{-\lambda^{q} s} Q(s) \omega(\lambda) d s \\
& =\int_{0}^{\infty} \int_{0}^{\infty} q t^{q-1} e^{-(\lambda t)^{q}} Q\left(t^{q}\right) e^{-\lambda s}(F x)(s) d s d t \\
& =\int_{0}^{\infty} \int_{0}^{\infty} \int_{0}^{\infty} q \psi_{q}(\theta) e^{-(\lambda t \theta)} Q\left(t^{q}\right) e^{-\lambda s} t^{q-1}(F x)(s) d \theta d s d t \\
& =\int_{0}^{\infty} \int_{0}^{\infty} \int_{0}^{\infty} q \psi_{q}(\theta) e^{-\lambda(t+s)} Q\left(\frac{t^{q}}{\theta^{q}}\right) \frac{t^{q-1}}{\theta^{q}}(F x)(s) d \theta d s d t \\
& =\int_{0}^{\infty} e^{-\lambda t} q \int_{0}^{t} \int_{0}^{\infty} \psi_{q}(\theta) Q\left(\frac{(t-s)^{q}}{\theta^{q}}\right) \\
& \left.\times \frac{(t-s)^{q-1}}{\theta^{q}}(F x)(s) d \theta d s\right] d t .
\end{aligned}
$$

According to (7) and (8), we have

$$
\begin{aligned}
\nu(\lambda)= & \int_{0}^{\infty} e^{-\lambda t}\left[q \int_{0}^{\infty} \psi_{q}(\theta) Q\left(\frac{t^{q}}{\theta^{q}}\right) \frac{t^{q-1}}{\theta^{q}}\left(x_{0}-g(x)\right) d \theta\right. \\
& \left.+q \int_{0}^{t} \int_{0}^{\infty} \psi_{q}(\theta) Q\left(\frac{(t-s)^{q}}{\theta^{q}}\right) \frac{(t-s)^{q-1}}{\theta^{q}}(F x)(s) d \theta d s\right] d t .
\end{aligned}
$$

Now we can invert the last Laplace transform to get

$$
\begin{aligned}
x(t)= & q \int_{0}^{\infty} \theta t^{q-1} M_{q}(\theta) Q\left(t^{q} \theta\right)\left(x_{0}-g(x)\right) d \theta \\
& +q \int_{0}^{t} \int_{0}^{\infty} \theta(t-s)^{q-1} M_{q}(\theta) Q\left((t-s)^{q} \theta\right)(F x)(s) d \theta d s \\
= & t^{q-1} P_{q}(t)\left(x_{0}-g(x)\right) \\
& +\int_{0}^{t}(t-s)^{q-1} P_{q}(t-s)(F x)(s) d s .
\end{aligned}
$$


The proof is complete.

Due to Lemma 3.3, we give the following definition of the mild solution of (1).

Definition 3.2. By the mild solution of the nonlocal Cauchy problem (1), we mean that the function $x \in C\left(J^{\prime}, X\right)$, which satisfies

$$
\begin{gathered}
x(t)=t^{q-1} P_{q}(t)\left(x_{0}-g(x)\right)+\int_{0}^{t}(t-s)^{q-1} P_{q}(t-s)(F x)(s) d s, \\
\text { for } t \in(0, a] .
\end{gathered}
$$

Suppose that $A$ is the infinitesimal generator of a strongly continuous semigroup of bounded linear operators (i.e., $C_{0}$ semigroup) $\{Q(t)\}_{t \geq 0}$ in Banach space $X$. This means that there exists $M>1$ such that $M=\sup _{t \in[0, \infty)}\|Q(t)\|_{B(X)}<\infty$.

Proposition 3.1 [19]. For any fixed $t>0,\left\{P_{q}(t)\right\}_{t>0}$ is linear and bounded operator, i.e., for any $x \in X$

$$
\left|P_{q}(t) x\right| \leq \frac{M}{\Gamma(q)}|x| .
$$

Proposition 3.2 [19]. Operator $\left\{P_{q}(t)\right\}_{t>0}$ is strongly continuous, which means that, for all $x \in X$ and $0<t^{\prime}<t^{\prime \prime} \leq a$, we have

$$
\left|P_{q}\left(t^{\prime \prime}\right) x-P_{q}\left(t^{\prime}\right) x\right| \longrightarrow 0 \quad \text { a.s. } t^{\prime \prime} \rightarrow t^{\prime}
$$

Proposition $3.3[\mathbf{1 9}]$. Assume that $\{Q(t)\}_{t>0}$ is a compact operator. Then $\left\{P_{q}(t)\right\}_{t>0}$ is also a compact operator.

4. Existence results. Define

$$
X^{(q)}\left(J^{\prime}\right)=\left\{x \in C\left(J^{\prime}, X\right): \lim _{t \rightarrow 0+} t^{1-q} x(t) \text { exists and is finite }\right\} .
$$


For any $x \in X^{(q)}\left(J^{\prime}\right)$, let the norm $\|\cdot\|_{q}$ be defined by

$$
\|x\|_{q}=\sup _{t \in(0, a]}\left\{t^{1-q}|x(t)|\right\} .
$$

Then $\left(X^{(q)}\left(J^{\prime}\right),\|\cdot\|_{q}\right)$ is a Banach space.

For $r>0$, define a closed subset $B_{r}^{(q)}\left(J^{\prime}\right) \subset X^{(q)}\left(J^{\prime}\right)$ as follows:

$$
B_{r}^{(q)}\left(J^{\prime}\right)=\left\{x \in X^{(q)}\left(J^{\prime}\right):\|x\|_{q} \leq r\right\} .
$$

Thus, $\left(B_{r}^{(q)}\left(J^{\prime}\right),\|\cdot\|_{q}\right)$ is a bounded, closed and convex subset of $X^{q}\left(J^{\prime}\right)$.

Let $B(J)$ be the closed ball of the space $C(J, X)$ with radius $r$ and center at 0 , that is,

$$
B(J)=\{y \in C(J, X):\|y\| \leq r\} .
$$

Thus $B(J)$ is a bounded, closed and convex subset of $C(J, X)$.

We introduce the following hypotheses.

$\left(H_{0}\right) Q(t)(t>0)$ is equicontinuous, i.e., $Q(t)$ is continuous in the uniform operator topology for $t>0$.

$\left(H_{1}\right)$ The operator $F: C\left(J^{\prime}, X\right) \rightarrow L\left(J^{\prime}, X\right)$ is continuous.

$\left(H_{2}\right)$ There exists a function $m \in L\left(J, \mathbf{R}^{+}\right)$such that

$$
I_{0+}^{q} m \in C\left(J^{\prime}, \mathbf{R}^{+}\right), \quad \lim _{t \rightarrow 0+} t^{1-q}\left(I_{0+}^{q} m\right)(t)=0,
$$

and

$|(F x)(t)| \leq m(t) \quad$ for all $x \in B_{r}^{(q)}\left(J^{\prime}\right)$ and almost all $t \in[0, a]$.

$\left(H_{3}\right)$ There exists a constant $L \in(0,(\Gamma(q) / M))$, the operator $g$ : $C\left(J^{\prime}, X\right) \rightarrow L\left(J^{\prime}, X\right)$ satisfies

$$
\left|g\left(x_{1}\right)-g\left(x_{2}\right)\right| \leq L\left\|x_{1}-x_{2}\right\|_{q}, \text { for } x_{1}, x_{2} \in B_{r}^{(q)}\left(J^{\prime}\right) .
$$

$\left(H_{4}\right)$ There exists a constant $r>0$ such that

$$
\frac{M}{\Gamma(q)-M L}\left(\left|x_{0}\right|+|g(0)|+\sup _{t \in(0, a]}\left\{t^{1-q} \int_{0}^{t}(t-s)^{q-1} m(s) d s\right\}\right) \leq r .
$$


$\left(H_{3}\right)^{\prime}$ The operator $g: C\left(J^{\prime}, X\right) \rightarrow L\left(J^{\prime}, X\right)$ is a continuous and compact map, and there exist positive constants $L_{1}, L_{2}$ such that $L_{1} \in(0,(\Gamma(q) / M))$ and $|g(x)| \leq L_{1}\|x\|_{q}+L_{2}$ for all $x \in B_{r}^{(q)}\left(J^{\prime}\right)$.

$\left(H_{4}\right)^{\prime}$ There exists a constant $r>0$ such that

$$
\frac{M}{\Gamma(q)-M L_{1}}\left(\left|x_{0}\right|+L_{2}+\sup _{t \in(0, a]}\left\{t^{1-q} \int_{0}^{t}(t-s)^{q-1} m(s) d s\right\}\right) \leq r
$$

For any $x \in B_{r}^{(q)}\left(J^{\prime}\right)$, define an operator $T$ as follows

$$
(T x)(t)=\left(T_{1} x\right)(t)+\left(T_{2} x\right)(t)
$$

where

$$
\begin{array}{ll}
\left(T_{1} x\right)(t)=t^{q-1} P_{q}(t)\left(x_{0}-g(x)\right), & \text { for } t \in(0, a], \\
\left(T_{2} x\right)(t)=\int_{0}^{t}(t-s)^{q-1} P_{q}(t-s)(F x)(s) d s, & \text { for } t \in(0, a] .
\end{array}
$$

It is easy to see that $\lim _{t \rightarrow 0+} t^{1-q}(T x)(t)=\left(x_{0}-g(x)\right) /(\Gamma(q))$. For any $y \in B(J)$, set

$$
x(t)=t^{q-1} y(t), \quad \text { for } t \in(0, a] .
$$

Then, $x \in B_{r}^{(q)}\left(J^{\prime}\right)$. Define $\mathscr{T}$ as follows:

$$
\left(\mathscr{T}_{y}\right)(t)=\left(\mathscr{T}_{1} y\right)(t)+\left(\mathscr{T}_{2} y\right)(t)
$$

where

$$
\begin{aligned}
& \left(\mathscr{T}_{1} y\right)(t)= \begin{cases}t^{1-q}\left(T_{1} x\right)(t), & \text { for } t \in(0, a], \\
\left(x_{0}-g(x)\right) /(\Gamma(q)), & \text { for } t=0,\end{cases} \\
& \left(\mathscr{T}_{2} y\right)(t)= \begin{cases}t^{1-q}\left(T_{2} x\right)(t), & \text { for } t \in(0, a], \\
0, & \text { for } t=0 .\end{cases}
\end{aligned}
$$

Obviously, $x$ is a mild solution of $(1)$ in $B_{r}^{(q)}\left(J^{\prime}\right)$ if and only if the operator equation $x=T x$ has a solution $x \in B_{r}^{(q)}\left(J^{\prime}\right)$. Before giving the main results, we firstly prove the following lemmas. 
Lemma 4.1. Assume that there exists a constant $r>0$, such that the conditions $\left(H_{1}\right)-\left(H_{4}\right)$ are satisfied. Then $T$ maps $B_{r}^{(q)}\left(J^{\prime}\right)$ into $B_{r}^{(q)}\left(J^{\prime}\right)$, and $T$ is continuous in $B_{r}^{(q)}\left(J^{\prime}\right)$.

Proof. Step I. T maps $B_{r}^{(q)}\left(J^{\prime}\right)$ into $B_{r}^{(q)}\left(J^{\prime}\right)$. For any $x \in B_{r}^{(q)}\left(J^{\prime}\right)$ and $t \in(0, a]$, by using $\left(H_{1}\right)-\left(H_{4}\right)$, we have

$$
\begin{aligned}
t^{1-q}|(T x)(t)| \leq & \left|P_{q}(t)\left(x_{0}-g(x)\right)\right| \\
& +t^{1-q}\left|\int_{0}^{t}(t-s)^{q-1} P_{q}(t-s)(F x)(s) d s\right| \\
\leq & \frac{M}{\Gamma(q)}\left(\left|x_{0}\right|+L\|x-0\|_{q}+|g(0)|\right) \\
& +\frac{M}{\Gamma(q)} t^{1-q} \int_{0}^{t}(t-s)^{q-1}|(F x)(s)| d s \\
\leq & \frac{M}{\Gamma(q)}\left(\left|x_{0}\right|+L r+|g(0)|\right. \\
& \left.+\sup _{t \in(0, a]}\left\{t^{1-q} \int_{0}^{t}(t-s)^{q-1} m(s) d s\right\}\right) \\
\leq & r .
\end{aligned}
$$

Hence, $\|T x\|_{q} \leq r$ for any $x \in B_{r}^{(q)}\left(J^{\prime}\right)$.

Step II. $T$ is continuous in $B_{r}^{(q)}\left(J^{\prime}\right)$. For any $x_{m}, x \in B_{r}^{(q)}\left(J^{\prime}\right)$, $m=1,2, \ldots$, with $\lim _{m \rightarrow \infty}\left\|x_{m}-x\right\|_{q}=0$, we get

$$
\lim _{m \rightarrow \infty} t^{1-q} x_{m}(t)=t^{1-q} x(t), \quad \text { for } t \in(0, a] .
$$

Then by condition $\left(H_{1}\right)$

$$
\sup _{t \in(0, a]}\left\{t^{1-q}\left|\left(F x_{m}\right)(t)-(F x)(t)\right|\right\} \longrightarrow 0 \quad \text { as } m \rightarrow \infty \text {. }
$$


On the other hand, for $t \in(0, a]$,

$$
\begin{aligned}
& t^{1-q}\left|\left(T x_{m}\right)(t)-(T x)(t)\right| \\
\leq & \left|P_{q}(t)\left(g\left(x_{m}\right)-g(x)\right)\right| \\
& +t^{1-q}\left|\int_{0}^{t}(t-s)^{q-1} P_{q}(t-s)\left(\left(F x_{m}\right)(s)-(F x)(s)\right) d s\right| \\
\leq & \frac{M L}{\Gamma(q)}\left\|x_{m}-x\right\|_{q} \\
& +\frac{M t^{1-q}}{\Gamma(q)} \int_{0}^{t}(t-s)^{q-1} s^{q-1} s^{1-q}\left|\left(F x_{m}\right)(s)-(F x)(s)\right| d s \\
\leq & \frac{M L}{\Gamma(q)}\left\|x_{m}-x\right\|_{q}+\frac{M a^{q} \Gamma(q)}{\Gamma(2 q)} \sup _{s \in(0, a]}\left\{s^{1-q}\left|\left(F x_{m}\right)(s)-(F x)(s)\right|\right\},
\end{aligned}
$$

which implies

$$
\left\|T x_{m}-T x\right\|_{q} \longrightarrow 0 \quad \text { as } m \rightarrow \infty \text {. }
$$

This means that $T$ is continuous in $B_{r}^{(q)}\left(J^{\prime}\right)$. The proof is complete.

Lemma 4.2. Assume that there exists a constant $r>0$ such that the conditions $\left(H_{1}\right)-\left(H_{4}\right)$ are satisfied. Then $\mathscr{T}_{2}$ maps $B(J)$ into $B(J)$ and $\mathscr{T}_{2}$ is continuous in $B(J)$.

Proof. Step I. $\mathscr{T}_{2}$ maps $B(J)$ into $B(J)$. For any $y \in B(J)$, we have

$$
\left|\left(\mathscr{T}_{2} y\right)(t)\right|=0 \leq r, \quad \text { for } t=0
$$

and

$$
\left|\left(\mathscr{T}_{2} y\right)(t)\right| \leq \frac{M}{\Gamma(q)} t^{1-q} \int_{0}^{t}(t-s)^{q-1} m(s) d s \leq r, \quad \text { for } t \in(0, a] .
$$

Hence, $\left\|\mathscr{T}_{2} y\right\|_{B} \leq r$, for any $y \in B(J)$.

Step II. $\mathscr{T}_{2}$ is continuous in $B(J)$. For any $y_{m}, y \in B(J), m=1,2, \ldots$, set $x_{m}(t)=t^{q-1} y_{m}(t)$ and $x(t)=t^{q-1} y(t)$ for $t \in(0, a]$. Thus, $x_{m}, x \in B_{r}^{(q)}(J)$. When $\lim _{m \rightarrow \infty}\left\|y_{m}-y\right\|_{B}=0$, we get

$$
\lim _{m \rightarrow \infty} y_{m}(t)=y(t), \quad \text { for } t \in[0, a],
$$


then

$$
\lim _{m \rightarrow \infty} t^{1-q} x_{m}(t)=t^{1-q} x(t), \quad \text { for } t \in(0, a] .
$$

Hence,

$$
\lim _{m \rightarrow \infty} \sup _{t \in(0, a]}\left\{t^{1-q}\left|\left(T_{2} x_{m}\right)(t)-\left(T_{2} x\right)(t)\right|\right\}=0 .
$$

On the other hand, for $t \in[0, a]$

$$
\left|\left(\mathscr{T}_{2} y_{m}\right)(t)-\left(\mathscr{T}_{2} y\right)(t)\right|= \begin{cases}t^{1-q}\left|\left(T_{2} x_{m}\right)(t)-\left(T_{2} x\right)(t)\right|, & \text { for } t \in(0, a] \\ 0, & \text { for } t=0 .\end{cases}
$$

Therefore,

$$
\left\|\mathscr{T}_{2} y_{m}-\mathscr{T}_{2} y\right\|_{B} \rightarrow 0 \quad \text { as } m \rightarrow \infty
$$

This means that $\mathscr{T}_{2}$ is continuous on $B(J)$. The proof is complete.

Lemma 4.3. Assume that $\left(H_{0}\right)-\left(H_{4}\right)$ hold. Then $\left\{\mathscr{T}_{2} y, y \in B(J)\right\}$ is equicontinuous.

Proof. For any $y \in B(J)$, for $t_{1}=0,0<t_{2} \leq a$, then, we get

$$
\begin{aligned}
\mid\left(\mathscr{T}_{2} y\right)\left(t_{2}\right)- & \left(\mathscr{T}_{2} y\right)(0) \mid \\
& =\left|t_{2}^{1-q} \int_{0}^{t_{2}}\left(t_{2}-s\right)^{q-1} P_{q}\left(t_{2}-s\right)(F x)(s) d s\right| \\
& \leq \frac{M}{\Gamma(q)} t_{2}^{1-q} \int_{0}^{t_{2}}\left(t_{2}-s\right)^{q-1} m(s) d s \longrightarrow 0 \text { as } t_{2} \rightarrow 0 .
\end{aligned}
$$


For $0<t_{1}<t_{2} \leq a$, we have

$$
\begin{aligned}
& \left|\left(\mathscr{T}_{2} y\right)\left(t_{2}\right)-\left(\mathscr{T}_{2} y\right)\left(t_{1}\right)\right| \\
\leq & \left|\int_{t_{1}}^{t_{2}} t_{2}^{1-q}\left(t_{2}-s\right)^{q-1} P_{q}\left(t_{2}-s\right)(F x)(s) d s\right| \\
& +\mid \int_{0}^{t_{1}} t_{2}^{1-q}\left(t_{2}-s\right)^{q-1} P_{q}\left(t_{2}-s\right)(F x)(s) d s \\
& \quad-\int_{0}^{t_{1}} t_{1}^{1-q}\left(t_{1}-s\right)^{q-1} P_{q}\left(t_{2}-s\right)(F x)(s) d s \mid \\
& +\mid \int_{0}^{t_{1}} t_{1}^{1-q}\left(t_{1}-s\right)^{q-1} P_{q}\left(t_{2}-s\right)(F x)(s) d s \\
& \quad-\int_{0}^{t_{1}} t_{1}^{1-q}\left(t_{1}-s\right)^{q-1} P_{q}\left(t_{1}-s\right)(F x)(s) d s \mid \\
\leq & \frac{M}{\Gamma(q)}\left|\int_{t_{1}}^{t_{2}} t_{2}^{1-q}\left(t_{2}-s\right)^{q-1} m(s) d s\right| \\
& +\frac{M}{\Gamma(q)} \int_{0}^{t_{1}}\left[t_{1}^{1-q}\left(t_{1}-s\right)^{q-1}-t_{2}^{1-q}\left(t_{2}-s\right)^{q-1}\right] m(s) d s \\
& +\left|\int_{0}^{t_{1}} t_{1}^{1-q}\left(t_{1}-s\right)^{q-1}\left[P_{q}\left(t_{2}-s\right)(F x)(s)-P_{q}\left(t_{1}-s\right)(F x)(s)\right] d s\right| \\
\leq & I_{1}+I_{2}+I_{3},
\end{aligned}
$$

where

$$
\begin{aligned}
I_{1}= & \frac{M}{\Gamma(q)} \mid \int_{0}^{t_{2}} t_{2}^{1-q}\left(t_{2}-s\right)^{q-1} m(s) d s \\
& -\int_{0}^{t_{1}} t_{1}^{1-q}\left(t_{1}-s\right)^{q-1} m(s) d s \mid, \\
I_{2}= & \frac{2 M}{\Gamma(q)} \int_{0}^{t_{1}}\left[t_{1}^{1-q}\left(t_{1}-s\right)^{q-1}-t_{2}^{1-q}\left(t_{2}-s\right)^{q-1}\right] m(s) d s, \\
I_{3}= & \left|\int_{0}^{t_{1}} t_{1}^{1-q}\left(t_{1}-s\right)^{q-1}\left[P_{q}\left(t_{2}-s\right)-P_{q}\left(t_{1}-s\right)\right](F x)(s) d s\right| .
\end{aligned}
$$

One can deduce that $\lim _{t_{2} \rightarrow t_{1}} I_{1}=0$, since $I_{0+}^{q} m \in C\left(J^{\prime}, \mathbf{R}^{+}\right)$. Noting that

$$
\left[t_{1}^{1-q}\left(t_{1}-s\right)^{q-1}-t_{2}^{1-q}\left(t_{2}-s\right)^{q-1}\right] m(s) \leq t_{1}^{1-q}\left(t_{1}-s\right)^{q-1} m(s),
$$


and $\int_{0}^{t_{1}} t_{1}^{1-q}\left(t_{1}-s\right)^{q-1} m(s) d s$ exists $\left(s \in\left[0, t_{1}\right]\right)$, then by the Lebesgue dominated convergence theorem, we have

$$
\int_{0}^{t_{1}}\left[t_{1}^{1-q}\left(t_{1}-s\right)^{q-1}-t_{2}^{1-q}\left(t_{2}-s\right)^{q-1}\right] m(s) d s \longrightarrow 0 \quad \text { as } t_{2} \rightarrow t_{1},
$$

and one can deduce that $\lim _{t_{2} \rightarrow t_{1}} I_{2}=0$.

In order for $\varepsilon>0$ to be small enough, we have

$$
\begin{aligned}
I_{3} \leq & \int_{0}^{t_{1}-\varepsilon} t_{1}^{1-q}\left(t_{1}-s\right)^{q-1}\left\|P_{q}\left(t_{2}-s\right)-P_{q}\left(t_{1}-s\right)\right\|_{B(X)}|(F x)(s)| d s \\
& +\int_{t_{1}-\varepsilon}^{t_{1}} t_{1}^{1-q}\left(t_{1}-s\right)^{q-1}\left\|P_{q}\left(t_{2}-s\right)-P_{q}\left(t_{1}-s\right)\right\|_{B(X)}|(F x)(s)| d s \\
\leq & t_{1}^{1-q} \int_{0}^{t_{1}}\left(t_{1}-s\right)^{q-1} m(s) d s \\
& \times \sup _{s \in\left[0, t_{1}-\varepsilon\right]}\left\|P_{q}\left(t_{2}-s\right)-P_{q}\left(t_{1}-s\right)\right\|_{B(X)} \\
& +\frac{2 M}{\Gamma(q)} \int_{t_{1}-\varepsilon}^{t_{1}} t_{1}^{1-q}\left(t_{1}-s\right)^{q-1} m(s) d s \\
\leq & I_{31}+I_{32}+I_{33},
\end{aligned}
$$

where

$$
\begin{aligned}
I_{31}= & \frac{r \Gamma(q)}{M} \sup _{s \in\left[0, t_{1}-\varepsilon\right]}\left\|P_{q}\left(t_{2}-s\right)-P_{q}\left(t_{1}-s\right)\right\|_{B(X)} \\
I_{32}= & \frac{2 M}{\Gamma(q)} \mid \int_{0}^{t_{1}} t_{1}^{1-q}\left(t_{1}-s\right)^{q-1} m(s) d s \\
& -\int_{0}^{t_{1}-\varepsilon}\left(t_{1}-\varepsilon\right)^{1-q}\left(t_{1}-\varepsilon-s\right)^{q-1} m(s) d s \mid \\
I_{33}= & \frac{2 M}{\Gamma(q)} \int_{0}^{t_{1}-\varepsilon}\left[\left(t_{1}-\varepsilon\right)^{1-q}\left(t_{1}-\varepsilon-s\right)^{q-1}-t_{1}^{1-q}\left(t_{1}-s\right)^{q-1}\right] m(s) d s .
\end{aligned}
$$

By $\left(H_{0}\right)$, it is easy to see that $I_{31} \rightarrow 0$ as $t_{2} \rightarrow t_{1}$. Similar to the proof that $I_{1}$ and $I_{2}$ tend to zero, we get $I_{32} \rightarrow 0$ and $I_{33} \rightarrow 0$ as $\varepsilon \rightarrow 0$. Thus, $I_{3}$ tends to zero independently of $y \in B(J)$ as $t_{2} \rightarrow t_{1}$, $\varepsilon \rightarrow 0$. Therefore, $\left|\left(\mathscr{T}_{2} y\right)\left(t_{2}\right)-\left(\mathscr{T}_{2} y\right)\left(t_{1}\right)\right|$ tends to zero independently 
of $y \in B(J)$ as $t_{2} \rightarrow t_{1}$, which means that $\left\{\mathscr{T}_{2} y, y \in B(J)\right\}$ is equicontinuous. The proof is complete.

Lemma 4.4. Assume that there exists a constant $r>0$ such that the conditions $\left(H_{1}\right),\left(H_{2}\right),\left(H_{3}\right)^{\prime}$ and $\left(H_{4}\right)^{\prime}$ are satisfied. Then $T$ maps $B_{r}^{(q)}\left(J^{\prime}\right)$ into $B_{r}^{(q)}\left(J^{\prime}\right)$, and $T$ is continuous in $B_{r}^{(q)}\left(J^{\prime}\right)$.

Proof. For any $x \in B_{r}^{(q)}\left(J^{\prime}\right)$, by using $\left(H_{1}\right),\left(H_{2}\right),\left(H_{3}\right)^{\prime}$ and $\left(H_{4}\right)^{\prime}$, we have

$$
\begin{aligned}
t^{1-q}|(T x)(t)| \leq & \left|P_{q}(t)\left(x_{0}-g(x)\right)\right| \\
& +t^{1-q}\left|\int_{0}^{t}(t-s)^{q-1} P_{q}(t-s)(F x)(s) d s\right| \\
\leq & \frac{M}{\Gamma(q)}\left(\left|x_{0}\right|+L_{1} r+L_{2}\right) \\
& +\frac{M t^{1-q}}{\Gamma(q)} \int_{0}^{t}(t-s)^{q-1}|(F x)(s)| d s \\
\leq & \frac{M}{\Gamma(q)}\left(\left|x_{0}\right|+L_{1} r+L_{2}\right. \\
& \left.+\sup _{t \in(0, a]}\left\{t^{1-q} \int_{0}^{t}(t-s)^{q-1} m(s) d s\right\}\right) \\
\leq & r .
\end{aligned}
$$

Hence, $\|T x\|_{q} \leq r$ for any $x \in B_{r}^{(q)}\left(J^{\prime}\right)$. Using a similar argument to that used in the proof of Lemma 4.1, we know that $T$ is continuous in $B_{r}^{(q)}\left(J^{\prime}\right)$, by $\left(H_{1}\right)$ and $\left(H_{4}\right)^{\prime}$. The proof is complete.

Lemma 4.5. Assume that there exists a constant $r>0$ such that the conditions $\left(H_{1}\right),\left(H_{2}\right),\left(H_{3}\right)^{\prime}$ and $\left(H_{4}\right)^{\prime}$ are satisfied. Then $\mathscr{T}$ maps $B(J)$ into $B(J)$, and $\mathscr{T}$ is continuous in $B(J)$.

Proof. For any $y \in B(J)$, we have

$$
|(\mathscr{T} y)(t)| \leq \frac{\left|x_{0}\right|+L_{1} r+L_{2}}{\Gamma(q)} \leq r, \quad \text { for } t=0
$$


and

$$
|(\mathscr{T} y)(t)|=t^{1-q}|(T x)(t)| \leq r, \quad \text { for } t \in(0, a]
$$

Hence, $\|\mathscr{T} y\|_{B} \leq r$, for any $y \in B(J)$. Clearly, $\mathscr{T}_{1}$ is continuous in $B(J)$, by $\left(H_{3}\right)^{\prime}$. Using a similar argument to that used in the proof of Lemma 4.2 , we know that $\mathscr{T}$ is continuous in $B(J)$. The proof is complete.

Lemma 4.6. Assume that there exists a constant $r>0$ such that the conditions $\left(H_{0}\right)-\left(H_{2}\right),\left(H_{3}\right)^{\prime}$ and $\left(H_{4}\right)^{\prime}$ are satisfied. Then $\{\mathscr{T} y, y \in B(J)\}$ is equicontinuous.

Proof. For any $y \in B(J)$, for $t_{1}=0,0<t_{2} \leq a$, we get

$$
\begin{aligned}
\left|(\mathscr{T} y)\left(t_{2}\right)-(\mathscr{T} y)(0)\right| \leq \mid & P_{q}\left(t_{2}\right)\left(x_{0}-g(x)\right)-\frac{x_{0}-g(x)}{\Gamma(q)} \mid \\
& +\left|t_{2}^{1-q} \int_{0}^{t_{2}}\left(t_{2}-s\right)^{q-1} P_{q}\left(t_{2}-s\right)(F x)(s) d s\right| \\
\leq & \left|P_{q}\left(t_{2}\right)\left(x_{0}-g(x)\right)-\frac{x_{0}-g(x)}{\Gamma(q)}\right| \\
& +\frac{M}{\Gamma(q)} t_{2}^{1-q} \int_{0}^{t_{2}}\left(t_{2}-s\right)^{q-1} m(s) d s \\
\longrightarrow & 0 \text { as } t_{2} \rightarrow 0 .
\end{aligned}
$$

For any $y \in B(J)$ and $0<t_{1}<t_{2} \leq a$, we get

$$
\begin{aligned}
\left|(\mathscr{T} y)\left(t_{2}\right)-(\mathscr{T} y)\left(t_{1}\right)\right| \leq & \left|\left(\mathscr{T}_{1} y\right)\left(t_{2}\right)-\left(\mathscr{T}_{1} y\right)\left(t_{1}\right)\right| \\
& +\left|\left(\mathscr{T}_{2} y\right)\left(t_{2}\right)-\left(\mathscr{T}_{2} y\right)\left(t_{1}\right)\right| \\
\leq & \left|\left(P_{q}\left(t_{2}\right)-P_{q}\left(t_{1}\right)\right)\left(x_{0}-g(x)\right)\right| \\
& +I_{1}+I_{2}+I_{3},
\end{aligned}
$$

where $I_{1}, I_{2}$ and $I_{3}$ are defined as in the proof of Lemma 4.3. According to Proposition 3.2, we know that $\left|(\mathscr{T} y)\left(t_{2}\right)-(\mathscr{T} y)\left(t_{1}\right)\right|$ tends to zero independently of $y \in B(J)$ as $t_{2} \rightarrow t_{1}$, which means that $\{\mathscr{T} y, y \in$ $B(J)\}$ is equicontinuous. The proof is complete. 
4.1. The case where $Q(t)$ is compact. In the following, we suppose that the operator $A$ generates a compact $C_{0}$-semigroup $\{Q(t)\}_{t \geq 0}$ on $X$, that is, for any $t>0$, the operator $Q(t)$ is compact.

Theorem 4.1. Assume that $Q(t)(t>0)$ is compact. Furthermore, assume that there exists a constant $r>0$ such that conditions $\left(H_{1}\right)-\left(H_{4}\right)$ are satisfied. Then the nonlocal Cauchy problem (1) has at least one mild solution in $B_{r}^{(q)}\left(J^{\prime}\right)$.

Proof. Since $Q(t)(t>0)$ is compact, from [14, Theorem 2.3.2], $Q(t)$ $(t>0)$ is equicontinuous, which implies that $\left(H_{0}\right)$ is satisfied.

For any $x_{1}, x_{2} \in B_{r}^{(q)}\left(J^{\prime}\right)$, according to $\left(H_{3}\right)$, we have

$$
\begin{aligned}
t^{1-q}\left|\left(T_{1} x_{1}\right)(t)-\left(T_{1} x_{2}\right)(t)\right| & \leq \frac{M}{\Gamma(q)}\left|g\left(x_{1}\right)-g\left(x_{2}\right)\right| \\
& \leq \frac{M L}{\Gamma(q)}\left\|x_{1}-x_{2}\right\|_{q},
\end{aligned}
$$

which implies that $\left\|T_{1} x_{1}-T_{1} x_{2}\right\|_{q} \leq(M L / \Gamma(q))\left\|x_{1}-x_{2}\right\|_{q}$. Thus, we obtain that

$$
\alpha\left(T_{1}\left(B_{r}^{(q)}\left(J^{\prime}\right)\right)\right) \leq \frac{M L}{\Gamma(q)} \alpha\left(B_{r}^{(q)}\left(J^{\prime}\right)\right) .
$$

Next, we will show that, for any $t \in[0, a], V(t)=\left\{\left(\mathscr{T}_{2} y\right)(t), y \in\right.$ $B(J)\}$ is relatively compact in $X$. Obviously, $V(0)$ is relatively compact in $X$. Let $t \in(0, a]$ be fixed. For all $\varepsilon \in(0, t)$ and for all $\delta>0$, define an operator $\mathscr{T}_{\varepsilon, \delta}$ on $B(J)$ by the formula

$$
\begin{aligned}
\left(\mathscr{T}_{\varepsilon, \delta} y\right)(t)= & q t^{1-q} \\
& \times \int_{0}^{t-\varepsilon} \int_{\delta}^{\infty} \theta(t-s)^{q-1} M_{q}(\theta) Q\left((t-s)^{q} \theta\right)(F x)(s) d \theta d s \\
= & q t^{1-q} Q\left(\varepsilon^{q} \delta\right) \\
& \times \int_{0}^{t-\varepsilon} \int_{\delta}^{\infty} \theta(t-s)^{q-1} M_{q}(\theta) Q\left((t-s)^{q} \theta-\varepsilon^{q} \delta\right)(F x)(s) d \theta d s,
\end{aligned}
$$

where $x \in B_{r}^{(q)}\left(J^{\prime}\right)$. Then from the compactness of $Q\left(\varepsilon^{q} \delta\right)\left(\varepsilon^{q} \delta>0\right)$, we obtain that the set $V_{\varepsilon, \delta}(t)=\left\{\left(\mathscr{T}_{\varepsilon, \delta} y\right)(t), y \in B(J)\right\}$ is relatively 
compact in $X$ for all $\varepsilon \in(0, t)$ and for all $\delta>0$. Moreover, for every $y \in B(J)$, we have

$$
\begin{aligned}
\left|\left(\mathscr{T}_{2} y\right)(t)-\left(\mathscr{T}_{\varepsilon, \delta} y\right)(t)\right| \leq \mid & t^{1-q} \int_{0}^{t} \int_{0}^{\delta} \theta(t-s)^{q-1} \\
& \times M_{q}(\theta) Q\left((t-s)^{q} \theta\right)(F x)(s) d \theta d s \mid \\
& +\mid q t^{1-q} \int_{t-\varepsilon}^{t} \int_{\delta}^{\infty} \theta(t-s)^{q-1} \\
& \times M_{q}(\theta) Q\left((t-s)^{q} \theta\right)(F x)(s) d \theta d s \mid \\
\leq & q t^{1-q} \int_{0}^{t}(t-s)^{q-1} m(s) d s \int_{0}^{\delta} \theta M_{q}(\theta) d \theta \\
& +q M^{1-q} \int_{t-\varepsilon}^{t}(t-s)^{q-1} m(s) d s \int_{0}^{\infty} \theta M_{q}(\theta) d \theta \\
\leq & q M t^{1-q} \int_{0}^{t}(t-s)^{q-1} m(s) d s \int_{0}^{\delta} \theta M_{q}(\theta) d \theta \\
& +\frac{M}{\Gamma(q)} t^{1-q} \int_{t-\varepsilon}^{t}(t-s)^{q-1} m(s) d s \\
\longrightarrow & 0 \text { as } \varepsilon \rightarrow 0, \delta \rightarrow 0 .
\end{aligned}
$$

Therefore, there are relatively compact sets arbitrarily close to the set $V(t), t>0$. Hence, the set $V(t), t>0$ is also relatively compact in $X$. Therefore, $\left\{\left(\mathscr{T}_{2} y\right)(t), y \in B(J)\right\}$ is relatively compact by the Ascoli-Arzela theorem.

For any $\left\{x_{n}\right\} \subset B_{r}^{(q)}\left(J^{\prime}\right)$, set

$$
y_{n}(t)= \begin{cases}t^{1-q} x_{n}(t), & \text { if } t \in(0, a], \\ y_{n}(0+), & \text { if } t=0 .\end{cases}
$$

Then $\left\{y_{n}\right\} \subset B(J)$. We can find at least one sequence $\left\{\mathscr{T}_{2} y_{n_{m}}\right\}_{m=1}^{\infty}$ which is convergent. Hence,

$$
\lim _{m \rightarrow \infty} t^{1-q}\left(T_{2} x_{n_{m}}\right)(t)=\lim _{m \rightarrow \infty}\left(\mathscr{T}_{2} y_{n_{m}}\right)(t), \quad \text { for } t \in(0, a] .
$$

This means that $\left\{T_{2} x_{n_{m}}\right\}_{m=1}^{\infty}$ is convergent in $B_{r}^{(q)}\left(J^{\prime}\right)$. Therefore, $\left\{\left(T_{2} x\right)(t), x \in B_{r}^{(q)}\left(J^{\prime}\right)\right\}$ is relatively compact. Thus, we have 
$\alpha\left(T_{2}\left(B_{r}^{(q)}\left(J^{\prime}\right)\right)\right)=0$. By $(9)$, we have

$$
\begin{aligned}
\alpha\left(T\left(B_{r}^{(q)}\left(J^{\prime}\right)\right)\right) & \leq \alpha\left(T_{1}\left(B_{r}^{(q)}\left(J^{\prime}\right)\right)\right)+\alpha\left(T_{2}\left(B_{r}^{(q)}\left(J^{\prime}\right)\right)\right) \\
& \leq \frac{M L}{\Gamma(q)} \alpha\left(B_{r}^{(q)}\left(J^{\prime}\right)\right)
\end{aligned}
$$

Thus, the operator $T$ is an $\alpha$-contraction in $B_{r}^{(q)}\left(J^{\prime}\right)$. By Lemma 4.1, we know that $T$ is continuous. Hence, Lemma 2.2 shows that $T$ has a fixed point in $B_{r}^{(q)}\left(J^{\prime}\right)$. Therefore, the nonlocal Cauchy problem (1) has a mild solution in $B_{r}^{(q)}\left(J^{\prime}\right)$. The proof is complete.

Theorem 4.2. Assume that $Q(t)(t>0)$ is compact. Furthermore, assume that there exists a constant $r>0$ such that the conditions $\left(H_{1}\right)$, $\left(\mathrm{H}_{2}\right),\left(\mathrm{H}_{3}\right)^{\prime}$ and $\left(\mathrm{H}_{4}\right)^{\prime}$ are satisfied. Then the nonlocal Cauchy problem (1) has at least one mild solution in $B_{r}^{(q)}\left(J^{\prime}\right)$.

Proof. Since $Q(t)(t>0)$ is compact, from [14, Theorem 2.3.2], $Q(t)$ $(t>0)$ is equicontinuous, which implies that $\left(H_{0}\right)$ is satisfied. Then, by Lemmas 4.4 and 4.5 , we know that $T: B_{r}^{(q)}\left(J^{\prime}\right) \rightarrow B_{r}^{(q)}\left(J^{\prime}\right)$ is bounded and continuous, $\mathscr{T}: B(J) \rightarrow B(J)$ is bounded, continuous and $\{\mathscr{T} y, y \in B(J)\}$ is equicontinuous.

According to the argument of Theorem 4.1, we only need to prove that, for any $t \in J$, the set $V_{1}(t)=\left\{\left(\mathscr{T}_{1} y\right)(t), y \in B(J)\right\}$ is relatively compact in $X$. Obviously, $V_{1}(0)$ is relatively compact in $X$. Let $0<t \leq a$ be fixed. For all $\delta>0$, define an operator $\mathscr{T}_{1}^{\delta}$ on $B(J)$ by the formula

$$
\begin{aligned}
\left(\mathscr{T}_{1}^{\delta} y\right)(t) & =q \int_{\delta}^{\infty} \theta M_{q}(\theta) Q\left(t^{q} \theta\right)\left(x_{0}-g(x)\right) d \theta \\
& =q Q\left(t^{q} \delta\right) \int_{\delta}^{\infty} \theta M_{q}(\theta) Q\left(t^{q} \theta-t^{q} \delta\right)\left(x_{0}-g(x)\right) d \theta
\end{aligned}
$$

where $x \in B_{r}^{(q)}(J)$. From the compactness of $Q\left(t^{q} \delta\right)\left(t^{q} \delta>0\right)$, we obtain that the set $V_{1}^{\delta}(t)=\left\{\left(\mathscr{T}_{1}^{\delta} y\right)(t), y \in B(J)\right\}$ is relatively compact 
in $X$ for all $\delta>0$. Moreover, for any $y \in B(J)$, we have

$$
\begin{aligned}
\left|\left(\mathscr{T}_{1} x\right)(t)-\left(\mathscr{T}_{1}^{\delta} x\right)(t)\right| & =\left|q \int_{0}^{\delta} \theta M_{q}(\theta) Q\left(t^{q} \theta\right)\left(x_{0}-g(x)\right) d \theta\right| \\
& \leq q M\left(\left|x_{0}\right|+L_{1} r+L_{2}\right) \int_{0}^{\delta} \theta M_{q}(\theta) d \theta .
\end{aligned}
$$

Therefore, there are relatively compact sets arbitrarily close to the set $V_{1}(t), t>0$. Hence, the set $V_{1}(t), t>0$, is also relatively compact in $X$. Moreover, $\{\mathscr{T} y, y \in B(J)\}$ is uniformly bound by Lemma 4.5. Therefore, $\{(\mathscr{T} y)(t), y \in B(J)\}$ is relatively compact by Ascoli-Arzela theorem. Using a similar method as in the proof of Theorem 4.1, we get $\left\{(T x)(t), x \in B_{r}^{(q)}\left(J^{\prime}\right)\right\}$ is relatively compact. Thus, $\alpha\left(T\left(B_{r}^{(q)}(J)\right)\right)=0$. Hence, Lemma 2.2 shows that $T$ has a fixed point in $B_{r}^{(q)}(J)$, which means that the nonlocal Cauchy problem (1) has a mild solution. The proof is complete.

Remark 4.1. If $g$ is not a compact map, we use another method given in $[\mathbf{2 0}]$ to consider the following integral equations

$$
\begin{aligned}
x(t)= & t^{q-1} P_{q}\left(t+\frac{1}{n}\right)\left(x_{0}-g(x)\right) \\
& +\int_{0}^{t}(t-s)^{q-1} P_{q}(t-s)(F x)(s) d s, \quad t \in(0, a] .
\end{aligned}
$$

For any $n \in \mathbf{N}^{+}$, noticing that the operator $Q(1 / n)$ is compact, one can easily derive the relative compactness of $V(0)$ and $V(t)(t>0)$. Then, (10) has one mild solution in $B_{r}^{(q)}\left(J^{\prime}\right)$. By passing the limit, as $n \rightarrow \infty$, one obtains a mild solution of the nonlocal Cauchy problem (1). However, because $Q(t)$ is replaced by $Q(1 / n)$, one needs a more restrictive condition than $\left(H_{4}\right)^{\prime}$, such as

$\left(H_{4}\right)^{\prime \prime}$ There exists a constant $r>0$ such that

$$
\frac{M_{\varepsilon}}{\Gamma(q)}\left(\left|x_{0}\right|+L_{1} r+L_{2}+\sup _{t \in(0, a]}\left\{t^{1-q} \int_{0}^{t}(t-s)^{q-1} m(s) d s\right\}\right) \leq r,
$$

where $M_{\varepsilon}=\sup _{t \in[0, a+\varepsilon]}\|Q(t)\|_{B(X)}, \varepsilon$ is a small constant. 
Now, we consider the nonlocal Cauchy problem (2) and introduce the following hypotheses.

$\left(h_{1}\right)$ For each $t \in J^{\prime}$, the function $f(t, \cdot): X \rightarrow X$ is continuous and, for each $x \in C\left(J^{\prime}, X\right)$, the function $f(\cdot, x): J^{\prime} \rightarrow X$ is strongly measurable.

$\left(h_{2}\right)$ There exists a function $m \in L\left(J, \mathbf{R}^{+}\right)$such that

$$
I_{0+}^{q} m \in C\left(J^{\prime}, \mathbf{R}^{+}\right), \quad \lim _{t \rightarrow 0+} t^{1-q}\left(I_{0+}^{q} m\right)(t)=0
$$

and

$$
|f(t, x)| \leq m(t) \quad \text { for all } x \in B_{r}^{(q)}\left(J^{\prime}\right) \text { and almost all } t \in[0, a] .
$$

Corollary 4.1. Assume that $Q(t)(t>0)$ is compact. Furthermore, assume that there exists a constant $r>0$ such that the conditions $\left(h_{1}\right)$, $\left(h_{2}\right),\left(H_{3}\right)$ and $\left(H_{4}\right)$ are satisfied. Then the nonlocal Cauchy problem (2) has at least one mild solution in $B_{r}^{(q)}\left(J^{\prime}\right)$.

Proof. Let $(F x)(t)=f(t, x)$. According to $\left(h_{1}\right)$ and $\left(h_{2}\right), f(t, x)$ is Lebesgue integrable. From Bochner's theorem, it follows that $f(t, x)$ is Bochner integrable. Thus, $F: C\left(J^{\prime}, X\right) \rightarrow L\left(J^{\prime}, X\right)$ is continuous. By Theorem 4.1, the conclusion of Corollary 4.1 holds. The proof is complete.

Similarly, we have the following corollary.

Corollary 4.2. Assume that $Q(t)(t>0)$ is compact. Furthermore, assume that there exists a constant $r>0$ such that the conditions $\left(h_{1}\right)$, $\left(h_{2}\right),\left(H_{3}\right)^{\prime}$ and $\left(H_{4}\right)^{\prime}$ are satisfied. Then the nonlocal Cauchy problem (2) has at least one mild solution in $B_{r}^{(q)}\left(J^{\prime}\right)$.

Remark 4.2. Condition $\left(h_{2}\right)$ of Corollaries 4.1 and 4.2 can be replaced by the following condition.

$\left(h_{2}\right)^{\prime}$ There exist a constant $q_{1} \in(0, q)$ and $m \in L^{1 / q_{1}}\left(J, \mathbf{R}^{+}\right)$such that

$$
|f(t, x)| \leq m(t), \quad \text { for all } x \in B_{r}^{(q)}\left(J^{\prime}\right) \text { and almost all } t \in[0, a] .
$$


In fact, if $\left(h_{2}\right)^{\prime}$ holds, by using the Hölder inequality, we know that $\left(h_{2}\right)$ holds.

4.2. The case where $Q(t)$ is not compact. If $Q(t)$ is noncompact, we give an assumption as follows.

$\left(H_{5}\right)$ There exists $\ell \in L\left(J, \mathbf{R}^{+}\right)$such that $I_{0+}^{q} \ell$ exists and, for any bounded $D \subset X$,

$$
\alpha(F(D)) \leq \ell(t) \alpha(D), \quad \text { for almost every } t \in[0, a] .
$$

Theorem 4.3. Assume that there exists a constant $r>0$ such that the conditions $\left(H_{0}\right)-\left(H_{5}\right)$ are satisfied. Then the nonlocal Cauchy problem (1) has at least one mild solution in $B_{r}^{(q)}\left(J^{\prime}\right)$.

Proof. By Lemmas 4.2 and 4.3, we know that $\mathscr{T}_{2}: B(J) \rightarrow B(J)$ is bounded, continuous and $\left\{\mathscr{T}_{2} y, y \in B(J)\right\}$ is equicontinuous. Next, we will show that $\mathscr{T}_{2}$ is compact in a subset of $B(J)$.

For each bounded subset $B_{0} \subset B(J)$, set

$$
\mathscr{T}^{1}\left(B_{0}\right)=\mathscr{T}_{2}\left(B_{0}\right), \quad \mathscr{T}^{n}\left(B_{0}\right)=\mathscr{T}_{2}\left(\overline{\mathrm{co}}\left(\mathscr{T}^{n-1}\left(B_{0}\right)\right)\right), \quad n=2,3, \ldots
$$

Similar to $[\mathbf{1 7}]$, there exists a $k \in(0,1)$ such that

$$
\alpha\left(\mathscr{T}^{\hat{n}}\left(B_{0}(t)\right)\right) \leq k \alpha\left(B_{0}\right)
$$

Then, we know from Proposition 2.1, $\mathscr{T}^{\hat{n}}\left(B_{0}(t)\right)$ is bounded and equicontinuous. Thus, from Proposition 2.2, we have

$$
\alpha\left(\mathscr{T}^{\hat{n}}\left(B_{0}\right)\right)=\max _{t \in[0, a]} \alpha\left(\mathscr{T}^{\hat{n}}\left(B_{0}(t)\right)\right)
$$

Hence,

$$
\alpha\left(\mathscr{T}^{\hat{n}}\left(B_{0}\right)\right) \leq k \alpha\left(B_{0}\right) .
$$

Furthermore, by Lemma 2.1 , there exists a $\widehat{D} \subset B$ such that

$$
\alpha\left(\mathscr{T}_{2}(\widehat{D})\right)=0
$$


Define

$$
\widehat{B}_{r}^{(q)}\left(J^{\prime}\right)=\left\{x \in C\left(J^{\prime}, X\right): x(t)=t^{q-1} y(t), t \in(0, a], y \in \widehat{D}\right\}
$$

with the norm $\|\cdot\|_{q}$ defined by

$$
\|x\|_{q}=\sup _{t \in(0, a]}\left\{t^{1-q}|x(t)|\right\} .
$$

Then, $\|x\|_{q}=\|y\|_{B} \leq r$, which implies $\widehat{B}_{r}^{(q)}\left(J^{\prime}\right) \subseteq B_{r}^{(q)}\left(J^{\prime}\right)$. Since $\mathscr{T}_{2}(\widehat{D})$ is compact, using a similar method as in the proof of Theorem 4.1 , we get $T_{2}\left(\widehat{B}_{r}^{(q)}\left(J^{\prime}\right)\right)$ is compact, i.e.,

$$
\alpha\left(T_{2}\left(\widehat{B}_{r}^{(q)}\left(J^{\prime}\right)\right)\right)=0
$$

On the other hand, for any $x_{1}, x_{2} \in \widehat{B}_{r}^{(q)}\left(J^{\prime}\right)$ and $t \in(0, a]$, according to $\left(H_{3}\right)$, we have

$$
\begin{aligned}
t^{1-q}\left|\left(T_{1} x_{1}\right)(t)-\left(T_{1} x_{2}\right)(t)\right| & \leq \frac{M}{\Gamma(q)}\left|g\left(x_{1}\right)-g\left(x_{2}\right)\right| \\
& \leq \frac{M L}{\Gamma(q)}\left\|x_{1}-x_{2}\right\|_{q}
\end{aligned}
$$

which implies that $\left\|T_{1} x_{1}-T_{1} x_{2}\right\|_{q} \leq(M L / \Gamma(q))\left\|x_{1}-x_{2}\right\|_{q}$. Thus, we obtain that

$$
\alpha\left(T_{1}\left(\widehat{B}_{r}^{(q)}\left(J^{\prime}\right)\right)\right) \leq \frac{M L}{\Gamma(q)} \alpha\left(\widehat{B}_{r}^{(q)}\left(J^{\prime}\right)\right)
$$

By (11) and (12), we have

$$
\begin{aligned}
\alpha\left(T\left(\widehat{B}_{r}^{(q)}\left(J^{\prime}\right)\right)\right) & \leq \alpha\left(T_{1}\left(\widehat{B}_{r}^{(q)}\left(J^{\prime}\right)\right)\right)+\alpha\left(T_{2}\left(\widehat{B}_{r}^{(q)}\left(J^{\prime}\right)\right)\right) \\
& \leq \frac{M L}{\Gamma(q)} \alpha\left(\widehat{B}_{r}^{(q)}\left(J^{\prime}\right)\right) .
\end{aligned}
$$

Thus, operator $T$ is an $\alpha$-contraction in $\widehat{B}_{r}^{(q)}\left(J^{\prime}\right)$. By Lemma 4.1, we know that $T$ is continuous. Hence, Lemma 2.2 shows that $T$ has a fixed point in $\widehat{B}_{r}^{(q)}\left(J^{\prime}\right) \subset B_{r}^{(q)}\left(J^{\prime}\right)$. Therefore, the nonlocal Cauchy problem (1) has a mild solution in $B_{r}^{(q)}\left(J^{\prime}\right)$. The proof is complete. 
Theorem 4.4. Assume that there exists a constant $r>0$ such that the conditions $\left(H_{0}\right)-\left(H_{2}\right),\left(H_{3}\right)^{\prime},\left(H_{4}\right)^{\prime}$ and $\left(H_{5}\right)$ are satisfied. Then the nonlocal Cauchy problem (1) has at least one mild solution in $B_{r}^{(q)}\left(J^{\prime}\right)$.

Proof. Since $g(x)$ is compact and $P_{q}(t)$ is bounded, $\left\{\left(\mathscr{T}_{1} y\right)(t), y \in\right.$ $B(J)\}$ is relatively compact. For any $\left\{x_{n}\right\} \subset B_{r}^{(q)}\left(J^{\prime}\right)$, set

$$
y_{n}(t)= \begin{cases}t^{1-q} x_{n}(t), & \text { if } t \in(0, a] \\ y_{n}(0+), & \text { if } t=0 .\end{cases}
$$

Then $\left\{y_{n}\right\} \subset B(J)$. We can find at least one sequence where $\left\{\mathscr{T}_{1} y_{n_{m}}\right\}_{m=1}^{\infty}$ is convergent. Hence,

$$
\lim _{m \rightarrow \infty} t^{1-q}\left(T_{1} x_{n_{m}}\right)(t)=\lim _{m \rightarrow \infty}\left(\mathscr{T}_{1} y_{n_{m}}\right)(t), \quad \text { for } t \in(0, a] .
$$

This means that $\left\{T_{1} x_{n_{m}}\right\}_{m=1}^{\infty}$ is convergent in $B_{r}^{(q)}\left(J^{\prime}\right)$. Therefore, $\left\{\left(T_{1} x\right)(t), x \in B_{r}^{(q)}\left(J^{\prime}\right)\right\}$ is relatively compact. Thus, we have $\alpha\left(T_{1}\left(B_{r}^{(q)}\left(J^{\prime}\right)\right)\right)=0$.

By the proof of Theorem 4.3, we know that there exists a $\widehat{B}_{r}^{(q)}\left(J^{\prime}\right) \subset$ $B_{r}^{(q)}\left(J^{\prime}\right)$ such that $T_{2}\left(\widehat{B}_{r}^{(q)}\left(J^{\prime}\right)\right)$ is relatively compact, i.e., $\alpha\left(T_{2}\left(\widehat{B}_{r}^{(q)}\left(J^{\prime}\right)\right)\right)$ $=0$. Hence, we have

$$
\alpha\left(T\left(\widehat{B}_{r}^{(q)}\left(J^{\prime}\right)\right)\right) \leq \alpha\left(T_{1}\left(\widehat{B}_{r}^{(q)}\left(J^{\prime}\right)\right)\right)+\alpha\left(T_{2}\left(\widehat{B}_{r}^{(q)}\left(J^{\prime}\right)\right)\right)=0 .
$$

Therefore, Lemma 2.2 shows that $T$ has a fixed point in $\widehat{B}_{r}^{(q)}\left(J^{\prime}\right) \subset$ $B_{r}^{(q)}\left(J^{\prime}\right)$. Therefore, the nonlocal Cauchy problem (1) has a mild solution in $B_{r}^{(q)}\left(J^{\prime}\right)$. The proof is complete.

Corollary 4.3. Assume that there exists a constant $r>0$ such that the conditions $\left(H_{0}\right),\left(h_{1}\right),\left(h_{2}\right)$ and $\left(H_{3}\right)-\left(H_{5}\right)$ are satisfied. Then the nonlocal Cauchy problem (2) has at least one mild solution in $B_{r}^{(q)}\left(J^{\prime}\right)$.

Corollary 4.4. Assume that there exists a constant $r>0$ such that the conditions $\left(H_{0}\right),\left(h_{1}\right),\left(h_{2}\right),\left(H_{3}\right)^{\prime},\left(H_{4}\right)^{\prime}$ and $\left(H_{5}\right)$ are satisfied. Then the nonlocal Cauchy problem (2) has at least one mild solution in $B_{r}^{(q)}\left(J^{\prime}\right)$. 
5. An example. Let $X=L^{2}([0, \pi], \mathbf{R})$. Consider the following fractional partial differential equations.

$$
\begin{cases}\partial_{t}^{q} u(t, z)=\partial_{z}^{2} u(t, z)+\partial_{z} G(t, u(t, z)), & z \in[0, \pi], t \in(0, a], \\ u(t, 0)=u(t, \pi)=0, & t \in(0, a], \\ u(0, z)+\sum_{i=0}^{n} \int_{0}^{\pi} k(z, y) u\left(t_{i}, y\right) d y=u_{0}(z), & z \in[0, \pi]\end{cases}
$$

where $\partial_{t}^{q}$ is a Riemann-Liouville fractional partial derivative of order $0<q<1, a>0, G$ is a given function, $n$ is a positive integer, $0<t_{0}<t_{1}<\cdots<t_{n} \leq a, u_{0}(z) \in X=L^{2}([0, \pi], \mathbf{R}), k(z, y) \in$ $L^{2}\left([0, \pi] \times[0, \pi], \mathbf{R}^{+}\right)$.

We define an operator $A$ by $A v=v^{\prime \prime}$ with the domain

$$
\begin{aligned}
& D(A)=\left\{v(\cdot) \in X: v, v^{\prime}\right. \text { absolutely continuous, } \\
& \left.\qquad v^{\prime \prime} \in X, v(0)=v(\pi)=0\right\} .
\end{aligned}
$$

Then $A$ generates a strongly continuous semigroup $\{Q(t)\}_{t \geq 0}$ which is compact, analytic and self-adjoint. Clearly, the nonlocal Cauchy problem $(2)$ and $\left(h_{1}\right)$ are satisfied.

System (13) can be reformulated as the following nonlocal Cauchy problem in $X$

$$
\left\{\begin{array}{l}
{ }^{L} D^{q} x(t)=A x(t)+f(t, x(t)), \quad \text { almost all } t \in[0, a], \\
\left(I_{0+}^{1-q} x\right)(0)+g(x)=x_{0}
\end{array}\right.
$$

where $x(t)=u(t, \cdot)$, that is, $x(t)(z)=u(t, z), t \in(0, a], z \in[0, \pi]$. The function $f: J^{\prime} \times X \rightarrow X$ is given by

$$
f(t, x(t))(z)=\partial_{z} G(t, u(t, z)),
$$

and the operator $g: C\left(J^{\prime}, X\right) \rightarrow L\left(J^{\prime}, X\right)$ is given by

$$
g(x)(z)=\sum_{i=0}^{n} K_{g} x\left(t_{i}\right)(z)
$$

where

$$
K_{g} v(z)=\int_{0}^{\pi} k(z, y) v(y) d y
$$


for $v \in X=L^{2}([0, \pi], \mathbf{R}), z \in[0, \pi]$.

We can take $q=1 / 3$ and $f(t, x(t))=t^{-1 / 4} \sin x(t)$, and choose

$$
m(t)=t^{-1 / 4}, \quad L=(n+1)\left(\int_{0}^{\pi} \int_{0}^{\pi} k^{2}(z, y) d y d z\right)^{1 / 2}
$$

and

$$
r=\frac{M}{\Gamma(1 / 3)-M L}\left(\left|x_{0}\right|+g(0)+\frac{\Gamma(1 / 3) \Gamma(3 / 4)}{\Gamma(12 / 13)} a^{3 / 4}\right) .
$$

Then, $\left(h_{1}\right),\left(h_{2}\right),\left(H_{3}\right)$ and $\left(H_{4}\right)$ are satisfied (noting that $K_{g}: X \rightarrow X$ is completely continuous). According to Corollary 4.1, system (13) has a mild solution in $B_{r}^{(1 / 3)}((0, a])$, provided that $(M L / \Gamma(1 / 3))<1$.

\section{REFERENCES}

1. J. Banas̀ and K. Goebel, Measure of noncompactness in Banach spaces, Marcel Dekker Inc., New York, 1980.

2. D. Bothe, Multivalued perturbation of $m$-accretive differential inclusions, Israel. J. Math. 108 (1998), 109-138.

3. K. Deimling, Nonlinear functional analysis, Springer-Verlag, New York, 1985.

4. D.J. Guo, V. Lakshmikantham and X.Z. Liu, Nonlinear integral equations in abstract spaces, Kluwer Academic, Dordrecht, 1996.

5. H.-P. Heinz, On the behaviour of measure of noncompactness with respect to differentiation and integration of vector-valued functions, Nonl. Anal.: TMA 7 (1983), 1351-1371.

6. E. Hernandez, D. O'Regan and Krishnan Balachandran, On recent developments in the theory of abstract differential equations with fractional derivatives, Nonl. Anal. 73 (2010), 3462-3471.

7. A.A. Kilbas, H.M. Srivastava and J. Juan Trujillo, Theory and applications of fractional differential equations, Elsevier Science B.V., Amsterdam, 2006.

8. S. Kumar and N. Sukavanam, Approximate controllability of fractional order semilinear systems with bounded delay, J. Diff. Equat. 252 (2012), 6163-6174.

9. V. Lakshmikantham and S. Leela, Nonlinear differential equations in abstract spaces, Pergamon Press, New York, 1969.

10. K. Li, J. Peng and J. Jia, Cauchy problems for fractional differential equations with Riemann-Liouville fractional derivatives, J. Funct. Anal. 263 (2012), 476-510.

11. L. Liu, F. Guo, C. Wu and Y. Wu, Existence theorems of global solutions for nonlinear Volterra type integral equations in Banach spaces, J. Math. Anal. Appl. 309 (2005), 638-649.

12. F. Mainardi, P. Paraddisi and R. Gorenflo, Probability distributions generated by fractional diffusion equations, in Econophysics: An emerging science, J. Kertesz and I. Kondor, eds., Kluwer, Dordrecht, 2000. 
13. H. Mönch, Boundary value problems for nonlinear ordinary differential equations of second order in Banach spaces, Nonl. Anal.: TMA 4 (1980), 985-999.

14. A. Pazy, Semigroups of linear operators and applications to partial differential equations, in Appl. Math. Sci. 44, Springer-Verlag, Berlin, 1983.

15. X.B. Shu, Y.Z. Lai and Y.M. Chen, The existence of mild solutions for impulsive fractional partial differential equations, Nonl. Anal.: TMA 74 (2011), 2003-2011.

16. A.Z.-A.M. Tazali, Local existence theorems for ordinary differential equations of fractional order, in Ordinary and partial differential equations, Lect. Notes Math. 964 (1982), Springer, Dundee.

17. J. Wang, Y. Zhou and M. Fečkan, Abstract Cauchy problem for fractional differential equations, Nonl. Dynam. 71 (2013), 685-700.

18. R. Wang, D. Chen and T.J. Xiao, Abstract fractional Cauchy problems with almost sectorial operators, J. Diff. Equat. 252 (2012), 202-235.

19. Y. Zhou and F. Jiao, Nonlocal Cauchy problem for fractional evolution equations, Nonl. Anal.: RWA 11 (2010), 4465-4475.

20. L.P. Zhu and G. Li, Nonlocal differential equations with multivalued perturbations in Banach spaces, Nonl. Anal. 69 (2008), 2843-2850.

Department of Mathematics, Xiangtan University, Hunan 411105, P.R. CHINA

Email address: yzhou@xtu.edu.cn

Department of Mathematics, Xiangtan University, Hunan 411105, P.R. CHINA

Email address: luzhang_math@126.com

Department of Mathematics, Xiangtan University, Hunan 411105, P.R. China

Email address: shenxiaohui2010@yahoo.cn 